\title{
Treatment trials of Pseudomonas aeruginosa infection in quails
}

\author{
Jihan M. Badr ${ }^{1}$, A. S. E. D. Metwali ${ }^{2 *}$, Amal I. Yoseif ${ }^{1}$, M. M. Arafa $^{3}$ \\ ${ }^{1}$ Department of Research \& Diagnosis of Poultry Diseases, ${ }^{3}$ Deptartment of Biochemistry, Animal \\ Health Research Institute Dokki- Giza, Egypt \\ ${ }^{2}$ Department of Poultry Diseases, Faculty of Veterinary Medicine, Cairo University, Giza, Egypt.
}

\begin{abstract}
An evaluation of the efficacy of different treatment trials in controlling Pseudomonas aeruginosa infection in quail chicks was done. Two probiotics (Lactobacilli and active dry yeast) and two fluoroquinolone antibiotics (Ciprofloxacin \& ofloxacin) were used. Four-daysold quail chicks were infected orally with $0.2 \mathrm{ml}$ containing $3 \times 108 \mathrm{CFU} / \mathrm{ml}$ of Pseudomonas aeruginsa strain which proved to be highly pathogenic for quails and sensitive to both antibiotics mentioned above. After three days, infected quails were sorted out into treated and control groups. The fluoroquinolone treated groups were given either of the antibiotics in the drinking water at a dose level of $10 \mathrm{mg} / \mathrm{kg} \mathrm{BW}$ every $12 \mathrm{~h}$ for five successive days, while the probiotic treated groups received either and both of the probiotics at a dose level of $0.5 \mathrm{gm} / \mathrm{liter}$ daily till the end of the experiment. The results revealed that fluoroquinolones were highly effective in destroying the infectious agent. on the other hand, the general health condition and body functions were negatively affected. Histopathological changes in fluoroquinolone and probiotic treated groups were similar. Bacteriologically, the probiotics failed to remove pseudomonas from the infected internal organs. Probiotics proved to be effective in reducing mortalities, severity of infection, enhancement of liver and kidney functions up to almost normal values and improving quail immunity. Probiotics resulted in improvement of the haematological and biochemical parameters as well as total serum proteins and serum globulins. Dual administration of the lactobacilli and saccharomyces justified their synergistic probiotics effect against quail chick pseudomoniasis.
\end{abstract}

\begin{abstract}
Abbreviations: $\mathrm{ALT}=$ alanine aminotransferase; $\mathrm{AP}=$ alkaline phosphatase; $\mathrm{AST}=$ aspartate amintransferase; $\mathrm{B}=$ basophils; $\mathrm{CFU}=$ colony forming unit $\mathrm{CFU} / \mathrm{ml}=$ colony forming unit per millilitre; Cipro.= ciprofloxacin; $\mathrm{E}=$ eosinophils; gm/ $\mathrm{Liter}=$ gram per liter; $\mathrm{H}=$ heterophils; $\mathrm{Hb}$ concentration $=$ haemoglobin concentration; $\mathrm{Hb} \%=$ haemoglobin percentage; $\mathrm{L}=$ lymphocytes; Lact.=lactobacillus; L. acidophilus = lactobacillus acidophilus; L brevis= lactobacillus brevis; L. planterum= lactobacillus planterum; $\mathrm{M}=$ monocytes; $\mathrm{MIC}=$ minimum inhibitory concentration; $\mathrm{Of}=$ ofloxacin; $\mathrm{PCV}=$ packed cell volume; $\mathrm{pH}=$ hydrogen ion concentration; $\mathrm{Ps}=$ pseudomonas aeruginosa; $\mathrm{R}=$ resistant; $\mathrm{TLC}=$ total leucocytic count; $\mathrm{T}$. lymphocytes $=$ thymus dependent lymphocytes; $\mathrm{Y}=$ active dry yeast.
\end{abstract}

In the last decade, there was growing interest in the establishment of quail farming in Egypt due to the increased number of quail meat lovers and the existence of an alternative to go up with the amount of poultry meat per citizen. Quails are less susceptible to bacterial diseases in comparison to other poultry species even though quail pseudomoniasis represents a great threat to quail producing plants due to; severe economic losses in quails and the environmental hazard to other poultry

\footnotetext{
* Corresponding author. Tel.: +20 27952732; fax: +205716840

E-mail address: ased_metwali@yahoo.com (A. S. E. D. Metwali)
}

species exists (Younes et al., 1990; El-Shafii and El-Sayed, 1992; Badr and Nasef, 2004). Moreover, quail pseudomoniasis has a public health importance to human beings (Ghoneim et al., 1980).

Pseudomonas aeruginosa is one of the most predominant pseudomonas species affecting quails causing serious economic losses, high morbidity, high mortalities (Hafez et al., 1987; El-Shorbagy et al., 2001) Unfortunately, the microorganism is naturally resistant to most antibiotics and quickly develops resistance to the commonly used ones Nakae et al, (1997). Pseudomonas infection increased liver enzymes in serum of infected birds Mankarios, (1999). 
There is a global concern over the restriction of antibiotics use in poultry industry, hence, there should be an urgent need to another safe and yet economic alternate for the prevention and control of bacterial diseases. The present investigation has been carried out to assess the efficacy of fluoroquinolones (Ciprofloxacin and ofloxacin) and probiotics (Saccharomyces cervisae and lactobacilli) in controlling Pseudomonas aeruginosa in quail chicks and their role in the improvement of liver and kidney functions as well as haematological picture.

\section{Material and Methods}

Quail chicks. Experimental quails were collected as one-day-old, litter reared according to the recommendations of the producer. Randomly sacrificed quails proved their freedom from bacteria especially Pseudomonas aeruginosa.

Pseudomonas field strain. A field strain of Pseudomonas was isolated from broiler chickens suffering from septicaemia and high mortality. Morphological, cultural and biochemical identifications were carried out according to (ElShorbagy et al., 2001; Quinn et al., 2002). The field strain was identified as Pseudomonas aeruginosa Susceptibility testing of the field strain was carried out by disc diffusion technique according to (Koneman et al., 1992) using different antibiotic discs supplied by Difco (Table 2).

Probiotics. Two commercial probiotics was used in this study: (1) Active dry yeast (Saccharoimyces cervisiae) (obtained from Holw Elsham Company for Powder and Light Food Industry, 6th October City, Giza, Egypt).

AVI-BAC. Commercial lactobacill: combination (Lactobacillus acidophilus; L. acidophilus; L. brevis and L. planterum). AVI- BAC was obtained from Sure Pharmaceutica Company, Heliopolis, Cairo, Egypt and produced by Pro-Byn International Inc., Lombard, Illinois 60148 USA. Each probiotic was used as supplement in the drinking water at a dose level of $0.5 \mathrm{gm}$ Probiotic/L drinking water once daily when mortalities appeared till the end of the observation period.

Fluoroquinolones. Two fluoroquinlones including ciprofloxacin and ofloxacin were used: (1) Ciprofloxacin infusion solution as ciprofloxacin lactate produced by (Amriya for Pharma ceutical Industries, Alexandria- Egypt). (2) Occufloxin eye drops as ofloxacin produced by the (Nile Company for Pharmaceutical and Chemical Industries, CairoEgypt). Ciprofloxacin and ofloxacin were added as $10 \mathrm{mg} / \mathrm{kg} \mathrm{BW}$ every $12 \mathrm{~h}$ when mortalities started and for five consecutive days.

Pilot pathogenicity testing of Pseudomonas aeruginosa field strain to quail chicks. Two-dayold quails were inoculated subcutaneously with
$0.2 \mathrm{ml}$ of $3 \mathrm{X} 108 \mathrm{CFU} / \mathrm{ml}$ of Pseudomonas aeruginosa (Table 1). Quails were fed on starter commercial quail feed and allowed fresh hygienic drinking water. Signs, mortalities and lesions were followed up and reisolation has been carried out from the infected internal organs.

Experimental design. Two hundred and ten 4-dayold quail chicks were used. Thirty chicks were used as control negative (Control) throughout the experiment. The remainder (180 quail chicks) were inoculated orally with individual dose of $0.2 \mathrm{ml}$ of $3 \times 108 \mathrm{CFU} / \mathrm{ml}$ of Pseudomonas aeruginosa field strain (Kheir El-Din et al., 1986). Inoculated chicks were kept under observation for three days for the development of clinical infection expressed as signs, mortalities and the reisolation of the inoculated strain from the internal organs of the infected quails. At the $4^{\text {th }}$ day post-infection inoculated quails were grouped into six treated groups as follows:

Group (2): Infected non-treated group considered as control positive.

Group (3) and (4): In which infected quails were treated with ciprofloxacin and ofloxacin respectively in the drinking water for five successive days after which they were received non medicated water till the end of the experiment.

Group (5) and (6): In which infected quails were given with Lactobacillus preparation (AVIBAC) and active dry yeast respectively in the drinking water till the end of the experiment.

Group (7): In which infected quails were treated with a mixture of Lactobacillus and active dry yeast in the drinking water till the end of the experiment. Ten days post treatment survivor quails were sacrificed and blood samples were collected for haematological and serum biochemical analysis. Postmortem changes were recorded and different organs were collected aseptically for bacteriological and histopathological examinations.

Bacteriological examination. Reisolation and biochemical identification of Pseudomonas aeruginosa strain from different internal organs of infected quails were performed according to (Holt et al., 1996; Quinn et al., 2002).

Histopahtological examination. Kidney, lung, liver, spleen and intestine were collected and fixed in $10 \%$ formol saline solution. The collected samples were dehydrated, cleared and embedded in paraffin wax then the specimens were sectioned to 4 $\mu \mathrm{m}$ thickness and stained by Haemotoxylin and Eosin (Koneman and Fulmen, 1976).

Haemogram. Blood samples collected from all groups at the end of the experiment. Erythrocytic count was performed using improved Neuober haemocytometer and Natt Herrick diluting fluid. Determination of haemoglobin was performed as 
described by (Van Kempen and Zijlstra, 1961). The packed cell volume (PCV) was estimated according to (Coles, 1986). Red cell index were calculated according to (Schalm, 1975). Total leucocytic counts were performed using the improved Neuober haemocytometer with and Natt and Herrick solution as diluting fluid according to the method described by (Natt and Herrick, 1952). Blood films stained with Giemsa stain were prepared for the determination of differential leucocytic count.

Serum biochemical parameters. Serum samples collected from all groups at the end of the experiment. Aspartate and alanine aminotransferase (AST and ALT) activities were determined colorimetrically according to (Retiman and Framkel, 1957). Serum alkaline phospatase (AP) was determined according to (Henry, 1974). Albumin was determined according to (Doumas, 1971). Serum creatinine was determined according to (Henry, 1979). Serum calcium was determined according to (Gindler and King, 1972). Serum phosphatase was determined according to (Goldenberg, 1966) and serum globulin according to (Doumas and Bigs, 1972).

Statistical analysis: Data collected from haematological and serum biochemical results of different groups of quails were statistically analyzed for the mean and standard deviation of the mean. Methods of statistical analysis were performed according to (Sndecor and Cochran, 1967).

\section{Results}

The pathogenicity testing of isolated Pseudomonas aeruginosa in quails (Table 2) resulted in $100 \%$ mortalities among infected quails within 48 hours after subcutaneous infection. Lesions showed severe septicaemia. The inoculated field strain of Pseudomonas aeruginosa could be reisolated from all internal organs. The antibiotic susceptibility test of the used Pseudomonas aeruginosa field strain against ten commonly used antibiotics (Table 1) revealed its sensitivity to fluoroquinolone antibiotics (Ciprofloxacin and ofloxacin).

The results of the mortality rate in different treatment groups showed that the mortality rate in the first 3 days post-infection reached $17.2 \%$ and continued in the non-treated infected group (Group 2) up to $78.26 \%$ toward the end of the experiment accompanied with the re-isolation of pseudomonas field strain from all infected internal organs.

The results of the haemogram are presented in (Table 4, Fig. 17); the results of the serum biochemical constituents are presented in (Table 5, Fig. 18). The results of the serum protein pattern are presented in (Table 6, Fig. 19) and finally the results of the histopathological changes are presented in (Tables 7, 8, Fig. 1-16).

The haemogram of the infected non-treated quails revealed that there was a significant decrease

in erythrocyte count, haemoglabin and PCV however, there was an increase in total leucocytic count especially lymphocytic and heterophilic count compared to the non infected birds.

The haemogam of the infected non-treated quails revealed that there was no significant change in erythocytic count, haemoglobin (Table 5). However there was an increase the total leuocytic count accompanied with significant increase in the lymphocytic and heterophil count (Table 5).

Serum biochemical analysis of the infected quails revealed high significant increase in the ALT, AST, AP, uric acid and creatinine. On other hand, there was a decrease in both calcium and phosphorus levels. Decrease in the total protein, albumen and globulin values were also observed. The electrophoretic pattern of serum globulin showed a decrease in gamma globulin fraction.

The histopathological examination of lung tissue showed severe congestion, haemorrhages (Fig.1), thrombosis, fibrinous exudates, inflammatory cell infiltration mainly lymphocytes, heterophils, macrophages, epithelial hyperplasia of secondary and tertiary bronchioles with goblet cell activation and granulomatous structure (Fig. 2). The kidney showed congestion, intertubular haemorrhages (Fig. 5), degenerative changes mainly vascular degeneration and cloudy swelling (Fig. 6), inflammatory cells, cystic formation containing bluish non-cellular material with necrobiotic changes in renal tubules (Fig. 7).

The liver showed congestion of hepatic blood vessels, degenerative changes of hepatocyts and necrobiotic changes. The spleen revealed depletion and ellipsoids (Fig. 14). The large intestine showed epithelial hyperplasia of mucosa with cystic formation of goblet cells (Fig. 16), while caecal tonsils revealed epithelial hyperplasia with goblet cell activation.

However it was found that the treatment of the infected birds with either ciprofloxacin or ofloxacin resulted in the reduction of mortality rate from $(86.6 \%)$ in groups 2 and 3 to $(19.05 \%$ and $23.8 \%)$ respectively, with high reduction of the number of pseudomonas-positive birds from $(100 \%)$ to $(11.76 \%$ and $12.5 \%)$ respectively, accompanied with decrease in the reisolation rate of the inoculated field strain of pseudomonas from the different internal organs (Table 3 ).

The haemogram findings showed an increased RBCs, haemoglobin, PCV, TLC, lymphocyte and 
Table (1): Results of the sensitivity test of the Pseudmonas aeruginosa field strain.

\begin{tabular}{ccccc}
\hline $\begin{array}{c}\text { Type } \\
\text { of the } \\
\text { Antibiotic }\end{array}$ & $\begin{array}{c}\text { Disc } \\
\text { concentration } \\
(\boldsymbol{\mu g})\end{array}$ & $\begin{array}{c}\text { Standered zone of } \\
\text { inhibition }(\geq \mathbf{m m})\end{array}$ & $\begin{array}{c}\text { Resulted zone of } \\
\text { inhibition } \mathbf{~ m m}\end{array}$ & Comment \\
\hline $\begin{array}{c}\text { Ampicillin } \\
\text { Amoxicillin }\end{array}$ & 20 & 14 & 0 & $\mathrm{R}$ \\
Chloramphenicol & 30 & 17 & 0 & $\mathrm{R}$ \\
Ciprofloxacin & 30 & 18 & 8 & $\mathrm{R}$ \\
Erythromycin & 5 & 22 & 25 & $\mathrm{~S}$ \\
Flumequin & 15 & 13 & 0 & $\mathrm{R}$ \\
Gentamycin & 30 & 19 & 0 & $\mathrm{R}$ \\
Neomycin & 10 & 16 & 0 & $\mathrm{R}$ \\
Ofloxacin & 30 & 14 & 23 & $\mathrm{R}$ \\
Tetracycline & 5 & 22 & 8 & $\mathrm{~S}$ \\
\hline
\end{tabular}

$\mathrm{R}=$ resistant $\mathrm{S}=$ sensitive

Table (2): Pilot pathogenicity test of Pseudomonas aeruginosa in quail Chicks.

\begin{tabular}{|c|c|c|c|c|c|c|c|c|c|c|c|c|c|}
\hline \multirow[t]{2}{*}{ Bacerial strain } & \multirow{2}{*}{$\begin{array}{c}\text { Dose of } \\
\text { infection } \\
\text { (CFU/ml) }\end{array}$} & \multirow[t]{2}{*}{$\begin{array}{l}\text { Route of } \\
\text { infection }\end{array}$} & \multirow{2}{*}{$\begin{array}{c}\text { No. of } \\
\text { infected } \\
\text { quails }\end{array}$} & \multirow[t]{2}{*}{$\begin{array}{c}\text { Age of } \\
\text { quails }\end{array}$} & \multicolumn{7}{|c|}{$\begin{array}{c}\text { Mortality/days post } \\
\text { infection }\end{array}$} & \multirow[t]{2}{*}{ Total } & \multirow[t]{2}{*}{$\%$} \\
\hline & & & & & 1 & 2 & 3 & 4 & 5 & 6 & 7 & & \\
\hline $\begin{array}{l}\text { Pseudomonas } \\
\text { aeruginosa }\end{array}$ & $6 \times 10^{7}$ & $\mathrm{~s} / \mathrm{c}$ & 10 & $\begin{array}{l}\text { 2-days } \\
\text { old }\end{array}$ & 8 & 2 & 0 & 0 & 0 & 0 & 0 & $10 / 10$ & $100 \%$ \\
\hline
\end{tabular}

Table (3): Reisolation rate of Pseudomonas aeruginosa from the different experimental groups.

\begin{tabular}{|c|c|c|c|c|c|c|c|c|c|c|c|}
\hline \multirow{2}{*}{$\begin{array}{l}\text { Gp. } \\
\text { No. } \\
\end{array}$} & \multirow[t]{2}{*}{ Treatment } & \multicolumn{2}{|c|}{ Liver } & \multicolumn{2}{|c|}{ Heart } & \multicolumn{2}{|c|}{ Lung } & \multicolumn{2}{|c|}{ Kidney } & \multicolumn{2}{|c|}{ Positive chicks } \\
\hline & & No. & $\%$ & No. & $\%$ & No. & $\%$ & No. & $\%$ & No. & $\%$ \\
\hline 1 & Control blank & 0 & 0 & 0 & 0 & 0 & 0 & 0 & 0 & 0 & 0 \\
\hline 2 & Ps. Infect & $5 / 5$ & 100 & $4 / 5$ & 80 & $5 / 5$ & 100 & $5 / 5$ & 100 & $5 / 5$ & 100 \\
\hline 3 & Ps. + Cipro. & $2 / 17$ & 11.76 & $0 / 17$ & 0 & $1 / 17$ & 5.88 & $0 / 17$ & 0 & $2 / 17$ & 11.76 \\
\hline 4 & Ps. + of. & $2 / 16$ & 12.5 & $1 / 16$ & 6.25 & $1 / 16$ & 6.25 & $2 / 16$ & 12.5 & $2 / 16$ & 12.5 \\
\hline 5 & Ps. + lact. & $13 / 15$ & 86.66 & $8 / 15$ & 53.33 & $10 / 15$ & 66.66 & $11 / 15$ & 73.33 & $13 / 15$ & 86.66 \\
\hline 6 & Ps. + Y. & $12 / 14$ & 85.71 & $9 / 14$ & 64.29 & $11 / 14$ & 78.57 & $12 / 14$ & 85.71 & $13 / 14$ & 92.86 \\
\hline 7 & Ps. + Lact. + Y. & $14 / 17$ & 82.35 & $7 / 17$ & 41.81 & $11 / 17$ & 64.71 & $12 / 17$ & 70.59 & $14 / 17$ & 82.35 \\
\hline
\end{tabular}

Ps. = Pseudomonas aerugionosa . Cipro. $=$ Ciprofloxacin.

Lact. $=$ Lactobacillus Preparation. Of. $=$ Ofloxacin. Y. $=$ Active dry yeast.

heterophils counts in ciprofloxacin, ofloxacin, lactobacilli and yeast treated as compared to pseudomonas infected non treated group. Serum biochemical analysis showed an increase in the AST, ALT, AP, creatinine and uric acid level

(Table 5). In addition, quails of the flouroquinolone treated groups $(3,4)$ showed no significant changes in their protein profiles (Table 8).

The histopathological findings in quails of groups 2, 4 showed a decrease in the lesion severity in all examined organs (Tables 7, 8, Fig. 8, 9).

As for as probiotics are concerned, the addition of lactobacilli or active dry yeast or both in the drinking water reduced the mortality rate to $28.57 \%, 33 \%$ and $19.05 \%$, respectively (Table 3 ) accompanied with the re-isolation of the inoculated pseudomonas strain from the different organs. The high reisolation rate was found in liver and kidney followed by lung. The results of the haemogram of lactobacilli treated and infected quails (Group 5) showed no significant changes in the RBC's count, $\mathrm{Hb} \%$, PCV. Significant increase in the TLC and lymphocytes \% was observed (Table 4). Serum biochemical analysis showed a transient increase in the AST and phosphorus levels with no significant changes noticed in other parameters (Table 5).

However the haemogram of the yeast treated group showed no changes in the RBC's count, $\mathrm{Hb} \%$, PCV and TLC (Table 4) and there were no significant differences in the serum biochemical parameters (Table 5), while the administration of both yeast and lactobacilli showed no significant effect on the RBC's count, $\mathrm{Hb} \%$, PCV with an 
Table (4): Haemogram of Pseudomonas aeruginosa infected quails and quails treated with probiotics and fluoroquinolones $(n=5)$.

\begin{tabular}{|c|c|c|c|c|c|c|c|c|c|c|c|c|}
\hline \multirow[t]{2}{*}{ Group } & \multirow{2}{*}{$\begin{array}{l}\text { RBCs } \\
10^{6} / \mu l\end{array}$} & \multirow{2}{*}{$\begin{array}{c}\mathrm{Hb} \\
\mathrm{gm} / \mathrm{dl}\end{array}$} & \multirow{2}{*}{$\begin{array}{c}\text { PCV } \\
\%\end{array}$} & \multirow{2}{*}{$\begin{array}{c}\mathrm{MCV} \\
\text { fl }\end{array}$} & \multirow{2}{*}{$\begin{array}{c}\text { MCH } \\
\text { pg }\end{array}$} & \multirow{2}{*}{$\begin{array}{c}\text { MCHC } \\
\text { g/dl }\end{array}$} & \multirow{2}{*}{$\begin{array}{c}\text { TLC } \\
10^{3} / \mu\end{array}$} & \multicolumn{5}{|c|}{ Differential WBCs } \\
\hline & & & & & & & & $\mathbf{E}$ & $\mathbf{B}$ & $\mathbf{M}$ & $\mathbf{L}$ & $\mathbf{H}$ \\
\hline \multirow[t]{3}{*}{ Control blank } & 3.2 & 11.5 & 34.40 & 107.5 & 35.9 & 33.3 & 2.8 & 0 & 0 & 9.2 & 22.0 & 68.8 \\
\hline & \pm & \pm & \pm & \pm & \pm & \pm & \pm & & & \pm & \pm & \pm \\
\hline & 0.32 & 0.95 & 0.95 & 1.24 & 0.86 & 0.71 & 0.9 & & & 0.04 & 1.03 & 0.61 \\
\hline \multirow{3}{*}{$\begin{array}{l}\text { Infected only } \\
\text { (control +ve) }\end{array}$} & 2.7 & 9.5 & 31.6 & 117.0 & 35.2 & 30.1 & 3.7 & 0 & 0 & 5 & 70.6 & 24.4 \\
\hline & \pm & \pm & \pm & \pm & \pm & \pm & \pm & & & \pm & \pm & \pm \\
\hline & 0.4 & 0.61 & 0.83 & 2.64 & 1.05 & 1.1 & 0.87 & & & 0.11 & 0.98 & 0.54 \\
\hline \multirow[t]{3}{*}{ Infected+cipro. } & 3.1 & 10.8 & 33.5 & 108.1 & 34.8 & 32.2 & 2.6 & 0 & 0 & 7.6 & 69.8 & 22.6 \\
\hline & \pm & \pm & \pm & \pm & \pm & \pm & \pm & & & \pm & \pm & \pm \\
\hline & 0.02 & 0.11 & 0.70 & 0.94 & 0.94 & 0.64 & 0.34 & & & 0.34 & 1.07 & 0.99 \\
\hline \multirow[t]{2}{*}{ Infected + of. } & $\begin{array}{c}3.66 \\
\pm\end{array}$ & $\begin{array}{c}12.2 \\
\pm\end{array}$ & $\begin{array}{c}36.8 \\
\pm\end{array}$ & $\begin{array}{c}100.5 \\
\pm\end{array}$ & $\begin{array}{c}33.3 \\
\pm\end{array}$ & $\begin{array}{c}33.2 \\
\pm\end{array}$ & $\begin{array}{c}3.6 \\
\pm\end{array}$ & 0 & 0 & $\begin{array}{c}5.6 \\
\pm\end{array}$ & $\begin{array}{c}74.4 \\
\pm\end{array}$ & $\begin{array}{c}20.0 \\
\pm\end{array}$ \\
\hline & 0.33 & 0.75 & 0.64 & 2.55 & 0.84 & 0.33 & 0.56 & & & 0.04 & 0.92 & 0.71 \\
\hline \multirow[t]{3}{*}{ Infected+yeast } & 3.6 & 12.5 & 37.7 & 104.7 & 34.7 & 33.2 & 3.52 & 0 & 0 & 2.4 & 72.6 & 25 \\
\hline & \pm & \pm & \pm & \pm & \pm & \pm & \pm & & & \pm & \pm & \pm \\
\hline & 0.09 & 0.42 & 0.74 & 2.7 & 1.03 & 0.98 & 0.9 & & & 0.06 & 0.94 & 0.7 \\
\hline \multirow[t]{2}{*}{ Infected+Lact } & $\begin{array}{c}3.6 \\
\pm\end{array}$ & $\begin{array}{c}11.4 \\
\pm\end{array}$ & $\begin{array}{c}36.4 \\
\pm\end{array}$ & $\begin{array}{c}101.1 \\
\pm\end{array}$ & $\begin{array}{c}31.6 \\
\pm\end{array}$ & $\begin{array}{c}31.3 \\
\pm\end{array}$ & $\begin{array}{c}3.1 \\
\pm\end{array}$ & 0 & 0 & $\begin{array}{c}4.2 \\
\pm\end{array}$ & $\begin{array}{c}74.6 \\
\pm\end{array}$ & $\begin{array}{c}21.2 \\
\pm\end{array}$ \\
\hline & 0.21 & 0.43 & 0.19 & 1.04 & 0.51 & 0.44 & 0.41 & & & 0.05 & 0.49 & 0.91 \\
\hline \multirow[t]{2}{*}{ Infected $+\mathbf{y}+\mathbf{L}$} & $\begin{array}{l}3.9 \\
+\end{array}$ & 13.2 & $\begin{array}{c}39.4 \\
+\end{array}$ & $\begin{array}{c}101.0 \\
+\end{array}$ & 33.8 & 33.5 & 3.8 & 0 & 0 & $\begin{array}{l}3.2 \\
+\end{array}$ & $\begin{array}{c}70.2 \\
+\end{array}$ & $\begin{array}{c}26.6 \\
+\end{array}$ \\
\hline & $\begin{array}{c} \pm \\
0.44\end{array}$ & $\begin{array}{c} \pm \\
0.61\end{array}$ & $\begin{array}{c} \pm \\
0.93\end{array}$ & $\begin{array}{c} \pm \\
0.91\end{array}$ & $\begin{array}{c} \pm \\
1.02\end{array}$ & $\begin{array}{c} \pm \\
0.9\end{array}$ & $\begin{array}{c} \pm \\
0.74\end{array}$ & & & $\begin{array}{c} \pm \\
0.01\end{array}$ & $\begin{array}{c} \pm \\
0.56\end{array}$ & $\begin{array}{c} \pm \\
0.84\end{array}$ \\
\hline
\end{tabular}

$\mathrm{E}=$ Eosinophils $\mathrm{B}=$ Basophils $\mathrm{Hb}=$ Haemoglobin

$\mathrm{M}=$ Monocytes $\mathrm{L}=$ Lymphocytes $\mathrm{H}=$ Heterophils

RBCs $=$ Erythrocytes PCV $=$ Packed cell volume

$\mathrm{MCV}=$ Mean corpuscular volume $\mathrm{MCH}=$ Mean corpuscular haemoglobin

$\mathrm{MCHC}=$ Mean corpuscular haemoglobin count. TLC $=$ Total leucocytic count.

Table (5): Serum biochemical constituents of Pseudomonas aeruginosa infected quails and quails treated with probiotics and Fluoroquinolones(n=5).

\begin{tabular}{|c|c|c|c|c|c|c|c|}
\hline Group & $\begin{array}{l}\text { ALT } \\
\mu / \mathrm{ml}\end{array}$ & $\begin{array}{l}\text { AST } \\
\mu / \mathrm{ml}\end{array}$ & $\begin{array}{c}\text { ALK.P } \\
\text { iu/l }\end{array}$ & $\begin{array}{c}\text { Creatinine } \\
\text { mg/dl }\end{array}$ & $\begin{array}{c}\text { Uric acid } \\
\mathrm{mg} / \mathrm{dl}\end{array}$ & $\begin{array}{c}\text { Calcium } \\
\mathrm{mg} / \mathrm{dl}\end{array}$ & $\begin{array}{c}\text { Phosphorus } \\
\text { mg/dl }\end{array}$ \\
\hline \multirow[t]{3}{*}{ Control blank } & 7.1 & 43.5 & 48.1 & 1.3 & 5.4 & 12.6 & 4.3 \\
\hline & \pm & \pm & \pm & \pm & \pm & \pm & \pm \\
\hline & 3.2 & 10.1 & 8.6 & 0.12 & 0.75 & 1.8 & 0.54 \\
\hline \multirow{3}{*}{$\begin{array}{l}\text { Infected only } \\
\text { (control +ve) }\end{array}$} & 12.2 & 64.1 & 70.5 & 1.9 & 8.1 & 9.2 & 3.1 \\
\hline & \pm & \pm & \pm & \pm & \pm & \pm & \pm \\
\hline & 1.7 & 4.2 & 5.1 & 0.16 & 0.75 & 1.9 & 0.78 \\
\hline \multirow[t]{3}{*}{ Infected+cipro. } & 14.3 & 65.2 & 77.1 & 2.1 & 8.9 & 9.6 & 3.9 \\
\hline & \pm & \pm & \pm & \pm & \pm & \pm & \pm \\
\hline & 2.9 & 4.3 & 4.9 & 0.3 & 0.9 & 1.2 & 0.64 \\
\hline \multirow[t]{3}{*}{ Infected + of. } & 19.6 & 68.1 & 75.2 & 2.2 & 9 & 9 & 3.9 \\
\hline & \pm & \pm & \pm & \pm & \pm & \pm & \pm \\
\hline & 3.3 & 6.7 & 8.4 & 0.33 & 0.64 & 1.55 & 0.64 \\
\hline \multirow[t]{3}{*}{ Infected+Lact } & 9.1 & 51.2 & 58.1 & 1.3 & 6.8 & 12 & 4.1 \\
\hline & \pm & \pm & \pm & \pm & \pm & \pm & \pm \\
\hline & 2.6 & 5.1 & 4.4 & 0.51 & 0.98 & 1.15 & 0.87 \\
\hline \multirow[t]{3}{*}{ Infected+yeast } & 8.1 & 50.1 & 60.2 & 1.5 & 7.1 & 11.2 & 3.9 \\
\hline & \pm & \pm & \pm & \pm & \pm & \pm & \pm \\
\hline & 1.6 & 4.3 & 5.6 & 0.42 & 0.91 & 1.03 & 0.9 \\
\hline \multirow[t]{3}{*}{ Infected $+\mathbf{y}+\mathbf{L}$} & 7.4 & 45.2 & 49 & 1.3 & 5.9 & 12.5 & 4.2 \\
\hline & \pm & \pm & \pm & \pm & \pm & \pm & \pm \\
\hline & 1.9 & 4.11 & 3.19 & 0.26 & 0.7 & 0.94 & 09 \\
\hline
\end{tabular}


Table (6): Serum protein pattern of Pseudomonas aeruginosa infected quails and quails treated with probiotics and fluoroquinolones $(n=5)$.

\begin{tabular}{cccccccc}
\hline Group & Total protein & $\begin{array}{c}\text { Albumin } \\
\text { gm/dl }\end{array}$ & $\begin{array}{c}\text { Total globulin } \\
\text { gm/dl }\end{array}$ & \multicolumn{4}{c}{ Globulin fraction } \\
\cline { 6 - 8 } & & & & Alpha & Beta & Gamma & A/G ratio \\
\hline Control blank & 3.4 & 1.2 & 2.2 & 0.4 & 0.5 & 1.3 & 0.54 \\
& \pm & \pm & \pm & \pm & \pm & \pm & \pm \\
Infected only & 0.39 & 0.36 & 0.3 & 0.02 & 0.04 & 0.2 & 0.13 \\
(control+ve) & 2.8 & 1.0 & 1.8 & 0.39 & 0.43 & 0.9 & 0.55 \\
& \pm & \pm & \pm & \pm & \pm & \pm & \pm \\
Infected+cipro. & 0.4 & 0.42 & 0.17 & 0.04 & 0.03 & 0.048 & 0.04 \\
& 3.05 & 1.02 & 2.03 & 0.41 & 0.51 & 1.1 & 0.49 \\
& \pm & \pm & \pm & \pm & \pm & \pm & \pm \\
Infected+of. & 0.16 & 0.54 & 1.4 & 0.03 & 0.013 & 0.11 & 0.05 \\
& 3 & 0.95 & 2.05 & 0.53 & 0.48 & 1.04 & 0.213 \\
Infected+Lact & \pm & \pm & \pm & \pm & \pm & \pm & \pm \\
& 0.43 & 0.56 & 0.16 & 0.01 & 0.04 & 0.003 & 0.11 \\
Infected+yeast & 3.5 & 1.0 & 2.5 & 0.60 & 0.53 & 1.37 & 0.4 \\
& \pm & \pm & \pm & \pm & \pm & \pm & \pm \\
& 0.62 & 0.33 & 0.9 & 0.05 & 0.05 & 0.16 & 0.05 \\
Infected+y+L & 3.4 & 1.6 & 2.8 & 0.50 & 0.55 & 1.75 & 0.57 \\
& \pm & \pm & \pm & \pm & \pm & \pm & \pm \\
& 0.79 & 0.99 & 0.33 & 0.03 & 0.06 & 0.71 & 0.12 \\
& 3.9 & 0.9 & 3 & 0.64 & 0.54 & 1.82 & 0.3 \\
& \pm & \pm & \pm & \pm & \pm & \pm & \pm \\
& 0.36 & 0.34 & 0.41 & 0.033 & 0.03 & 0.16 & 0.01 \\
\hline
\end{tabular}

Table (7): Histopathological findings recorded in lung and kidney of pseudomonas infected quails and quails treated with probiotics and fluoroquinolones.

\begin{tabular}{|c|c|c|c|c|c|c|c|c|c|c|c|c|c|c|}
\hline \multirow[b]{2}{*}{ Treatment } & \multicolumn{7}{|c|}{ * Lung lesions } & \multicolumn{7}{|c|}{ * Kidney lesions } \\
\hline & 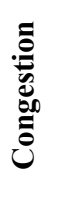 & 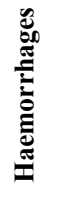 & 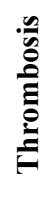 & 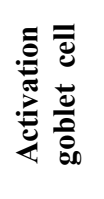 & & 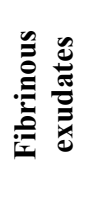 & 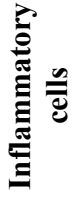 & 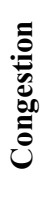 & 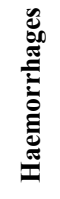 & 苞 & 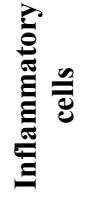 & 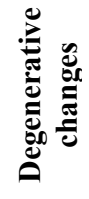 & פัّ & 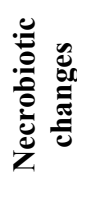 \\
\hline Control blank & - & - & - & - & - & - & - & - & - & - & - & - & - & - \\
\hline Pseud. Infection & 4 & 4 & 4 & 4 & 4 & 4 & 4 & 4 & 4 & 4 & 4 & 4 & 4 & 4 \\
\hline Pseud. ciprofloxacin & 2 & 3 & 2 & 3 & - & 2 & 2 & 3 & 2 & 2 & 2 & 3 & 3 & 3 \\
\hline Pseud. + ofloxacin & 3 & 3 & 2 & 3 & - & 3 & 4 & 2 & 2 & 1 & 2 & 2 & 2 & 2 \\
\hline Pseud. +lact. & 2 & 2 & 1 & 1 & - & 1 & 2 & 1 & 1 & 2 & 1 & 2 & 2 & 2 \\
\hline Pseud. +yeast & 2 & 3 & 2 & 2 & - & 3 & 3 & 2 & 2 & 3 & 2 & 2 & 3 & 3 \\
\hline Pseud. +lact. $+\mathbf{y}$ & 2 & 2 & 2 & 2 & - & 2 & 3 & 2 & 1 & 1 & 2 & 2 & 2 & 2 \\
\hline
\end{tabular}

* Histopathology score : (1)=Mild lesion. (2)=Pronounced lesion. (3)=Moderate lesion. (4)=Severe lesion. 
Table (8): Histopathological findings recorded in liver, spleen and intestine of Pseudomonas aeruginosa infected quails and infected treated quails with probiotics and fluoroquinolones.

\begin{tabular}{|c|c|c|c|c|c|c|c|c|c|}
\hline \multirow[b]{3}{*}{ Treatment } & \multirow{2}{*}{\multicolumn{3}{|c|}{ *Liver }} & \multirow{2}{*}{\multicolumn{2}{|c|}{ *Spleen }} & \multicolumn{4}{|c|}{ *Intestine } \\
\hline & & & & & & \multicolumn{2}{|c|}{ Large intestine } & \multicolumn{2}{|c|}{ Ceacal tonsils } \\
\hline & Congestion & $\begin{array}{l}\text { Degenerative } \\
\text { changes }\end{array}$ & $\begin{array}{l}\text { Necrobiotic } \\
\text { changes }\end{array}$ & Depletion & Ellipsoids & $\begin{array}{c}\text { Epithelial } \\
\text { hyperplasia }\end{array}$ & $\begin{array}{l}\text { Activation } \\
\text { goblet cell }\end{array}$ & $\begin{array}{c}\text { Epithelial } \\
\text { hyperplasia }\end{array}$ & $\begin{array}{l}\text { Activation } \\
\text { goblet cell }\end{array}$ \\
\hline Control blank & + & - & - & - & - & - & - & - & - \\
\hline $\begin{array}{l}\text { Pseudomonas } \\
\text { infection }\end{array}$ & 3 & 3 & 3 & 4 & 4 & 4 & 4 & 4 & 4 \\
\hline $\begin{array}{l}\text { Pseudomonas }+ \\
\text { ciprofloxacin }\end{array}$ & 2 & 2 & 2 & 2 & 2 & 2 & 1 & 2 & 2 \\
\hline $\begin{array}{l}\text { Pseudomonas }+ \\
\text { ofloxacin }\end{array}$ & 2 & 3 & 3 & 3 & 3 & 3 & 2 & 3 & 2 \\
\hline $\begin{array}{l}\text { Pseudomonas } \\
\text { +lactobacillus }\end{array}$ & 2 & 2 & 1 & 2 & 2 & 2 & 1 & 2 & 2 \\
\hline $\begin{array}{c}\text { Pseudomonas } \\
\text { +yeast }\end{array}$ & 2 & 1 & 1 & 2 & 2 & 1 & 2 & 1 & 1 \\
\hline $\begin{array}{c}\text { Pseudomonas } \\
\text { + lactobacilli } \\
\text { +yeast }\end{array}$ & 1 & 1 & 1 & 2 & 1 & 1 & 1 & 1 & 1 \\
\hline
\end{tabular}

Histonathologv score : (1)=Mild lesion. $(2)=$ Pronounced lesion. $(3)=$ Moderate lesion. $(4)=$ Severe lesion.

increase in TLC only (Table 4) and there was a transient increase in the ALT, AST, AP and creatinine values (Table 5). Also there were no significant changes in the uric acid, calcium and phosphorus levels (Table 5). There was a decrease in the albumen and an increase in the total globulins associated with significant increase in the $\mathrm{Y}$ globulins fraction in group treated with both yeast and lactobacilli (Table 6). The histopathological findings revealed a reduction in the degree of severity of the lesions compared to pseudomonas infected non treated group (Tables 7, 8, Fig. 3, 4, $10,11,12,13,15)$.

\section{Discussion}

Pseudomonas aeruginosa is widely distributed in nature and was formerly described as saprophytic microorganism (Koncicki and Szubstarska, 1988; Quinn et al., 2002; Raja Rajeswarl et al., 1992; Schildger et al., 1989; Younes et al., 1990) but recently it has been considered as an important cause of infection that has the ability to induce a disease among different animals and birds (Awaad, et al., 1981; Eisa, 1998; El-Shafii and El-Sayed 1992; El-Shorbagy et al., 2001; Hafez et al., 1987). This necessitates strict sanitary measures in the poultry husbandry especially feeds and water supply and the prevention of overcrowding and stress.

In the present study, pseudomonas induced septicaemia and high mortalities among the infected quail chicks. Similar observations were reported by (Younes et al., 1990; El-Shafii and El-Sayed, 1992) and agreed with El-Shorbagy et al., (2001) who reported that quails of all ages are susceptible to Pseudomonas aerugionosa with severe respiratory manifestations, dyspnea and profuse diarrhea.

Dead birds also suffered from septicaemia and this agreed with (Awaad, et al., 1981; Shihata et al., 1988; Raja Rajeswarl et al., 1992; El-Shorbagy et al., 2001). On the other hand, the results of the sensitivity test of fluoroquinolone antibiotic (Ciprofloxacin and ofloxacin), were similar to results obtained by (Osman et al., 1998; Tanios and Kamel, 1999; El-Shorbagy et al., 2001). The great variation in the results of the antibiotic sensitivity could be attributed to the uncontrolled use of antibiotics as a field practice and to the physiochemical properties of the cell wall rather the antibiotic inhibiting enzymes (Koncicki and Szubstarska, 1988).

The histopathological results proved that pseudomonas infection was accompanied with significant congestion, haemorrhages and thrombosis which may be due to severe septicaemia in all internal organs and similar results was also reported by (El-Shorbagy et al., 2001). The necrobiotic changes and degenerative changes in the different tissues indicated that the degree of damage which affected the organ cells mainly lung, liver 



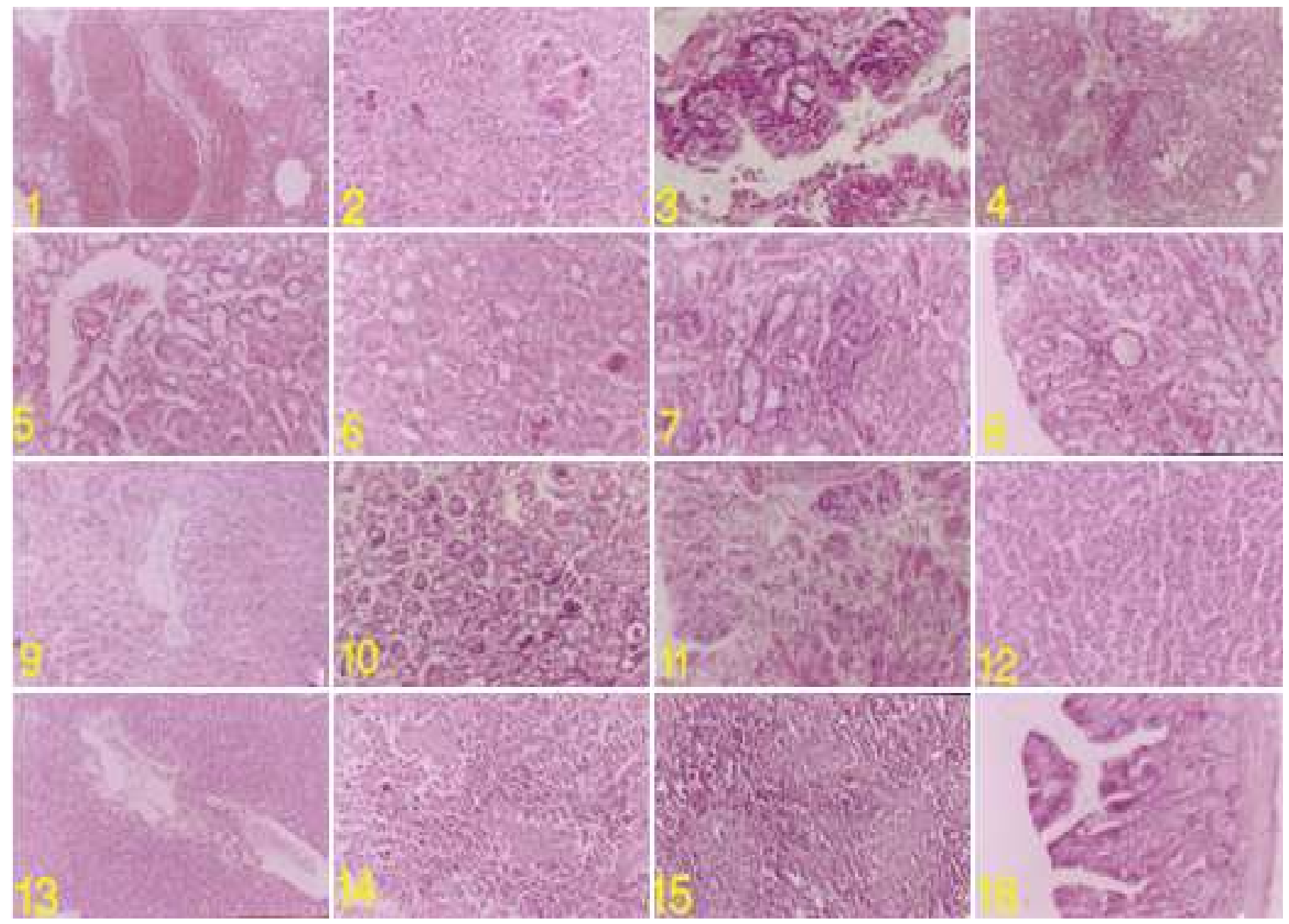

Fig.(1): Lung of infected quail showing severe haemorrhage (H\&E x 100).

Fig.(2): Lung of infected quail showing granuloma (H\&E x 250).

Fig.(3): Lung of yeast treated quail with yeast showing cystic formation of goblet cells (H\&E $x$ 250).

Fig.(4): Lung of yeast treated quail showing moderate haemorrhage (H\&E $x$ 100).

Fig.(5): Kidney of infected quail showing intertubular haemorrhage. (H\&E x 250).

Fig.(6): Kidney of infected quail showing cloudy swelling and vacuolar degeneration (H\&E $x$ 250).

Fig.(7): Kidney of infected quail showing cystic formation containing bluish non -cellular material, inflammatory cells infiltration and necrobiotic changes of renal tubules (H\&E $\times 250)$.

Fig.(8): Kidney of ciprofloxacin treated quail showing cystic formation containing bluish non - cellular material and necrobiotic changes of renal tubules (H\&E $\times 250$ ).

Fig.(9): Kidney of ciprofloxacin treated quail showing denudation, necrobiotic changes and haemorrhage (H\&E x 250).

Fig.(10): Kidney of yeast treated quail with showing mild denudation (H\&E $x$ 250).

Fig.(11): Kidney of yeast and lactobacillus treated quail showing mild denudation, necrobiotic changes and haemorrhage (H\&E $x$ 100).

Fig.(12): Liver of ciprofloxacin treated quail showing mild degenerative changes (H\&E x 250).

Fig.(13): Liver of ofloxacin treated quail showing thromosis (H\&E x 250).

Fig.(14): Spleen of infected quail showing depletion and ellipsoids (H\&E $\times 250$ ).

Fig.(15): Spleen of lactobacillus treated quail showing mild depletion (H\&E $x$ 250).

Fig.(16): Large intestine of infected quail showing cystic formation of goblet cells (H\&E x 250). 
Fig. (17): Haemogram of Pseudomonas aeruginosa infected quails and infected treated quails with probiotics and fluoroquinolones $(n=5)$.

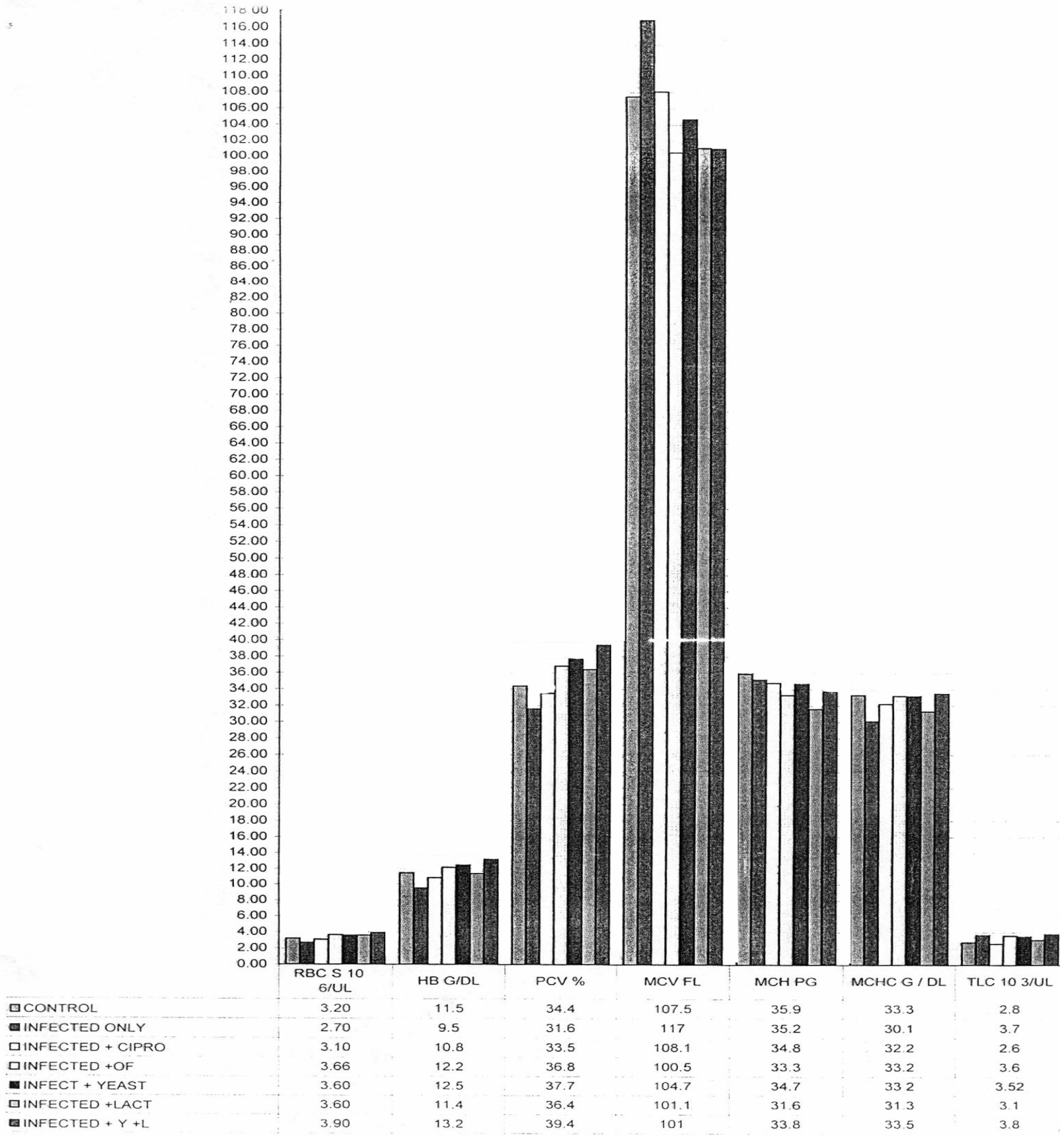


Fig. (18): Serum biochemical constituents of Pseudomonas aeruginosa infected quails and infected treated quails with probiotics and fluoroquinolones $(n=5)$.

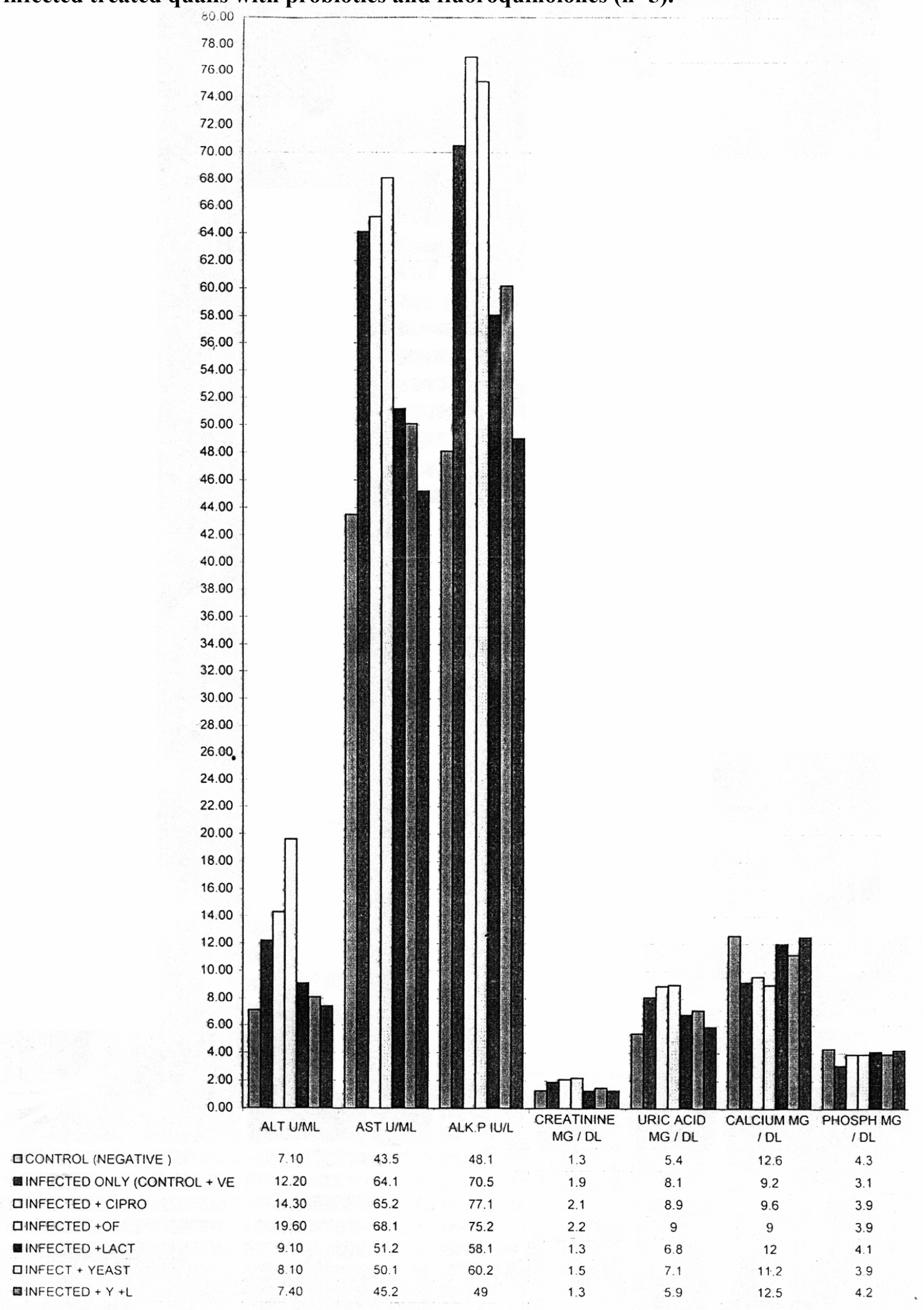


Fig. (19): Serum protein pattern of Pseudomonas aeruginosa infected quails and infected treated quails with probiotics and fluoroquinolones $(n=5)$.

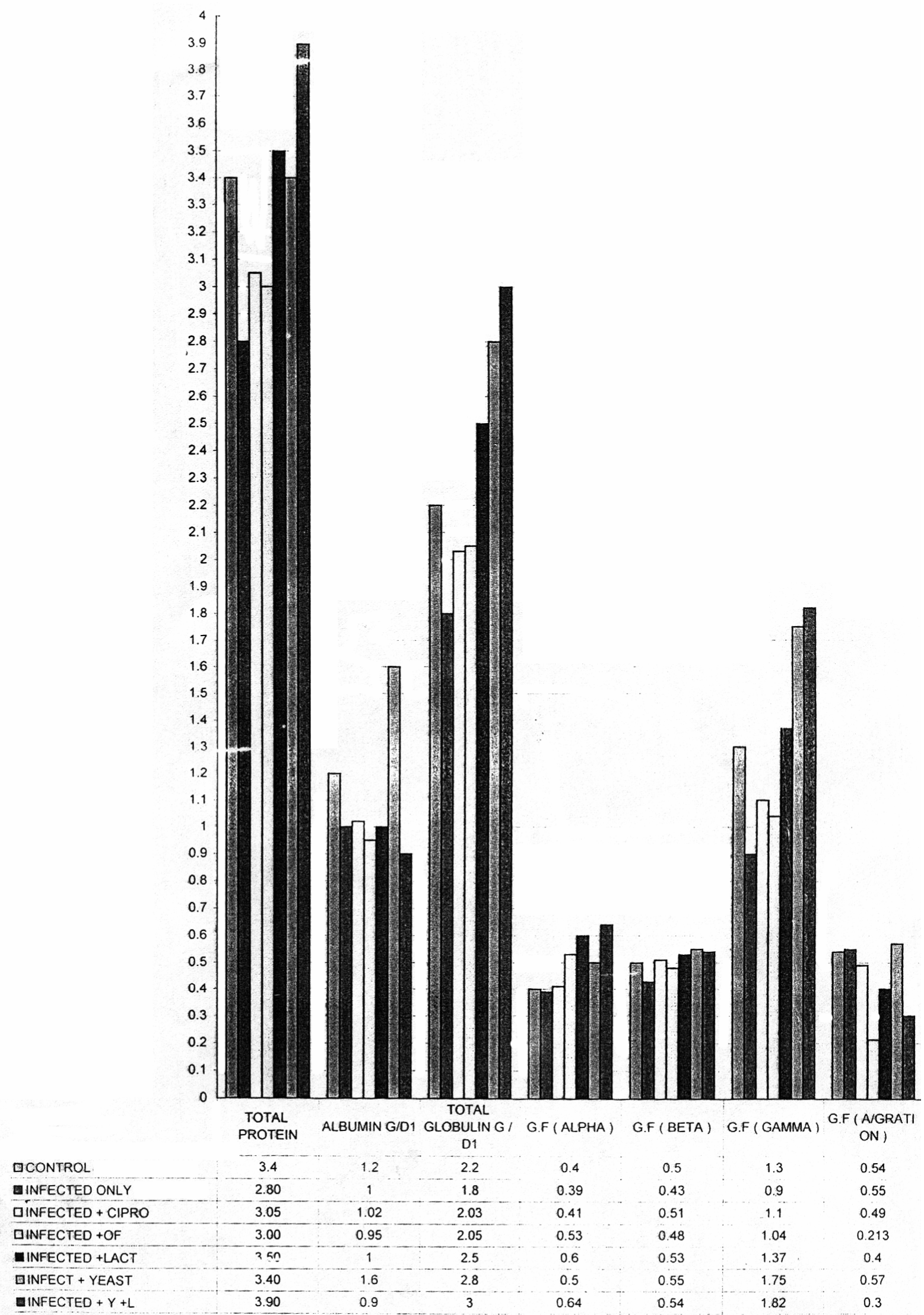


and kidney and this finding agree with (El-Shorbagy et al., 2001; Mazetti, 1972). Inflammatory cells infiltration mainly lymphocytes and heterophils in the different organs might be due to the antigenic stimulation of pseudomonas and this is backed up by the findings of Coles, (1986) and Hafez, et al., (1987). Depletion of lymphocytes in the spleen was in agreement with (Homes et al., 1978). The results of the biochemical and haematological examination proved that pseudomonas infection has been associated with significant decrease in RBC's count $\mathrm{Hb}$ concentration and $\mathrm{PCV} \%$ while there was an increased TLC (Lymphocytosis and heterophilia) which might be due to the antigenic stimulation of Pseudomonas aeruginosa which leads to an increase in the thymus dependent lymphocytes (Tlymphocytes) production as reported (Coles, 1986). Also, there was an elevation in the serum activities of ALT, AST and AP which seem probably to be due to the liver damage by the effect of Pseudomonas aeruginosa toxins following the escape of the enzymes into the serum in an abnormal high level (Eisa, 1998; Ross et al, 1976). The results were confirmed by the histopathological findings. There was also a significant decrease in the total proteins and albumin in the infected nontreated birds which might possibly be attributed to renal dysfunction (Hoe and Harvey, 1980; Mankarios, 1999) in addition to, the hypoalbuminaemia which is an important feature of the liver disease (Kaneko, 1980) as the liver is the sole site of albumin synthesis. There was an increase in the levels of creatinine, uric acid and decrease in calcium and phosphorus which is expected in birds with an impaired renal function (Halliwell, 1981; Hanafy, 1993). These could be attributed to the degenerative changes in the kidney tubules which prevent the excretion of creatinine and uric acid and lead to an increase in their level in the serum (Eisa, 1998; Kaneko, 1980). Nowadays ciprofloxacin and ofloxacin are newly introduced antibiotics in the veterinary practice(Siebert, 1988) and proved to be highly effective against wide range of infections caused by gram-negative bacteria (Cheesebrough et al., 1991; Rowe et al., 1991; Rowe et al., 1992). In the present study, it was found that treatment of birds with ciprofloxacin or ofloxacin in he drinking water at dose level of $10 \mathrm{mg} / \mathrm{Kg}$ live body weight led to a great reduction in the mortality rates, decrease in the number of pseudomonas positive birds and lowered the reisolation rate from different organs. As fluoroquinolone therapy has the advantages of low minimal inhibitory concentration (MIC) and high therapeutic concentration in serum, tissues and faeces after the oral administration (Goosen et al., 1985) accompanied with a prolonged excretion period which may persist for several days after therapy cessation, effectively prolongs the duration of the antibacterial activity Pequet et al., (1990) and this explains the significant reduction in the reisolation rate among fluoroquinolone treated groups.

Despite the fact that fluoroquinolone resistance is chromosomally mediated, yet the mutation can occur very rapidly within few days of the start of the treatment (Howard et al., 1990; Anon, 1992) and this explained the presence of small number of pseudomonas positive birds in the fluoroquinolone treated groups (Table 3).

Moreover, fluoroquinolone was accompanied with an increase in both creatinine and uric acid levels which could be attributed to the renal failure mostly due to the reduction of the glomerular filtration rate; similar results were obtained by Mankarios, (1999). There was a significant increase in AST, ALT in fluoroquinolone treated quails which might be attributed to the alterations in the membrane permeability or damage of hepatic cells by direct effect of the drug resulting in the escape of these enzymes to the plasma (Hanafy, 1993). These findings are supported by the results recorded by Osama, (1996). Meanwhile, the highly significant rise in the serum alkaline phosphatase (AP) associated with the fluoroquinolone therapy occurred after the hepatocellular damage which greatly agrees with the results of Eisa, (1998).

On the other hand, it was found that the treatment of the Pseudomonas infected quails with lactobacilli or active dry yeast or both at dose level of $0.5 \mathrm{gm} /$ litre in the drinking water was associated with high decline in he mortality rate, slight reduction in the reisolation rate; pseudomonas positive birds and decrease in the severity of lesions. In spite of the occurrence of the infection, it was found that all of the tested biochemical parameters tend to be normal in probiotic treated birds accompanied with an increase in both cellular and humoral immune responses of the infected birds.

Generally the effect of probiotics against enteropathogenic bacteria is introduced through the reduction of the intestinal colonization (Czerucka et al., 1994) through competitive exclusion by competing with the pathogenic microorganism for the adhesion sites or nutritional source (Barnhart et al., 1999; Bernet et al., 1994; Pascula et al., 1999)or by the reduction in caecal hydrogen ion concentration (Caecal $\mathrm{pH}$ ) especially in case of lactobacilli administration (Barnhart et al., 1999; 
Ezz El-Deen; ZoulFakar, 2003). In this study; the colonization, organ invasion and the infection already happened and the possible effect of the probiotics might be through the inhibition of the production or the action of the bacterial toxins (Ezz El-Deen and ZoulFakar, 2003; Brandao et al., 1998) and also through the stimulation of the systemic immune response by lactobacilli (Huang et al., 2004 ; Quere and Girard, 1999) or by yeast polysaccharides which were proved to be very promising immuno-stimulating agent that increases both humoral and cell mediated immune responses against pathogenic bacteria (Jing, et al., 1998; Badr and El-Kholy, 2003). It was reported that the effect of probiotic could be influenced by the type of the biotherapeutic agent, the method of administration, the viability of the preparation, the condition of the host and the gut microflora (Fuller, 1995). In addition, the specificity of the protective mechanism could be other factor that affects the action of the used probiotic (Filho-Lima et al., 2000) and this might explain the synergistic effect of both lactobacilli and active dry yeast in controlling the severity of pseudomonas infection in quails.

In conclusion, fluoroquinolone antibiotics are effective in the treatment of pseudomonas infection in quails but severely affect organs function, biochemical parameters and immunity of the treated birds. The production of mutant strains of the pathogenic bacteria resists the effect of the antibiotics. The use of the probitoic is effective in decreasing the severity of infection, enhancement of the body functions of the bird to overcome the disturbances occurring due to by the invading bacteria and enhanced both humoral and cellular immune responses of the bird. Active dry yeast and lactobacilli proved to have a synergistic effect in controlling Pseudomonas aeruginosa infection in quails.

\section{References}

Anon (1992): Compylobacter jejuni resistant to ciprofloxacin Communicable disease review, 26, 1 cited in Evaluation of combined antibiotic and competitive exclusion treatment in broiler breeder flocks infected with Salmonella enterica serovar enteritidis. By D.J. Reynolds; R.H. Davis; M. Richards and C. Wray. Avain pathol. 26: 38-95.

Awaad, M. H. H.; Youssef, H. I.; Saad, F. E. and Sorrakbi, T. M. B. (1981): Studies on Pseudomonas in chickens. Vet. Med. J. 29: 135-143.

Badr, J. M. and El-Kholy, M. M. (2003): Some studies on yeast-supplemented ration in salmonella infected layer chicks. J. Egypt. Vet. Med. Assoc. 63 (5): 23-36.

Badr, J. M. and Nasef, S. A. (2004): The role of free-living wild birds in transmitting certain bacterial infections to poultry. Egypt. J. Vet. Med. Assoc. 64 (2): 79-91.

Barnhart, E. T.; Caldwell, D. J..; Crouch, M. C..; Byrd, J. A.; Carrier, D. E. and Hargis, B. M. (1999): Effect of lactose administration in drinking water prior to and during feed withdrawal on salmonella recovery from broiler crops and caeca. Poult. Sci. 78: 211-214.
Bernet, M. F.; Brassart, D.; Neeser, J. R. and Sevin, A. L. (1994): Lactobacillus acidophilus LA1 binds to human intestinal cell lines and inhibits cell attachment and cell invasion by enterovirulent bacteria. Gut. 35: 483-489.

Brandao, R. L.; Castro, I. M. and Bambirra, E. A. (1998): Intracellular signal triggered by cholera toxin in Saccharomyes boulardii and Saccharomyes cerevisiae. App. Env. Microbiol. 64: 546-568.

Cheesebrough, J. S.; Mwema, F. I..; Green, S. D. R. and Tilloston, G. S. (1993): Quinolones in children with invasive salmonellosis. Lancet. 333: 127.

Coles, E. H. (1986): Veterinary Clinical Pathology.4th ed. W.B. Sounders Company, Philadelphia, London, Toronto, Mexico, Sydney, Tokyo, Hong Kong.

Czerucka, D.; Roux, I. and Rampal, P. (1994): Saccharomyes boulardii inhibits Secretory gut-mediated adenosine 3,5-cyclic monophosphate induction in intestinal cells. Gastroenterol. 106: 65-72.

Doumas, B. (1971): Colorimeteric determination of serum albumin. Clin. Chem. Acta: pp. 400-403.

Doumas, B. T. and Bigs, H. G. (1972): Determination of serum globulin. In: Standard Methods of Clinical chemistry, Vol. 7, ed. G. R. Cooper. New York Academic press.

Eisa A. M. (1998): Clinicopathological studies on some antidiarrhoeal drugs in rabbits. M.V.Sc. Thesis, Fac. Vet. Med., Zagazig Univ., Zagazig. Egypt.

El-Shafii, S. and El-Sayed, A. (1992): Studies on pseudomonas infection in poultry in Kalioubia Province. M.V.Sc., Thesis, Microbiology, Moshtohor Fac. Vet. Med., Zagazig Univ. (Banha branch) Elkalioubia, Egypt.

El-Shorbagy, M. A.; Mosaad, A. A.; Hussein, B. M. and Abd. Allah, M. M. (2001): Studies on Pseudomonas aeruginosa infection in quails. J. Egypt. Vet. Med. Ass. 61 (1): 205-221.

Ezz El-Deen, N. A. and ZoulFakar, S A. (2003): Role of lactic acid and/or garlic in controlling colonization and shedding of Salmonella typhimurium in chicks. J. Egypt. Vet. Med. Assoc. 63 (1): 75, 86.

Filho-Lima, J. V. M.; Vieira, E. C., and Nicoli, J. R. (2000): Antagonistic effect of Lactobacillus acidophilus, Saccharomyes boulardii and Escherichia coli combinations against experimental infection with Shigella flexneri and Salmonella enteritidis subsp. Typhimurium in gnotobiotic mice. J. Appl. Microbiol. 88: 365-370.

Fuller, R. (1995): Probiotics: Their development and use. in Probiotics: Prospects of use in opportunistic infections eds. R, Fuller.; P.J, Heidt.; V, Rusch and D. Van Der Waaji. p.1-8. Hebron Institute, for Microbiology.

Ghoneim, N. H.; Taha, N. L.; and Siam, M. A. (1980): Possible role of quails (Cortunix delegorguei) in the epidemiology of some bacterial infections with reference to an occupational case. Vet. Med. J., xxvIII (28): 295.

Gindler, G. M. and King, J. D. (1972): Rapid determination of calcium in biological fluid with methyl-thymol blue. Am.J. Clin. Path. 58, 376.

Goldenberg, H. (1966): Determination of serum phosphorus. Clin. Chem. 12: 871.

Goosen, H.; Demol, P.; Corgnau, H. J.; Levy, H.; Grados, O. and Ghysels, G. (1985): Comparative in vitro activities of aztreonorm, ciprofloxacin, norofloxacin, ofloxacin,HR810 (A new cephalosporin), RV28905 (A new macrolide) and other agents against enteropathogens. Antimicrob. Agents and Chemotherapy. 271: 388-392.

Hafez, H. M.; Woorrak, H. and Heil, G. (1987): Pseudomonas infection in turkey poults and treatment trials with apramycin. Berliner and Münchner Tierärzltiche Wochen Schrift. 100 (2):48-51.

Halliwell, W. H. (1981): Serum chemistry profiles in health and diseases of birds of preg. in recent advances In The Study 
of raptor diseases, Eds. J.E. Cooper Green and A.G. wood. Chion. Publication LTd. West York Shire, England.

Hanafy A. M. A. (1993): Some adverse effects of norfloxacin in male rats. M.V.Sc. Thesis, Fac. Vet. Med., Edfina, Alexandria Univ. Beheraa, Egypt.

Henry, R. J. (1974): Colorimeteric method for determination of serum total protein, Clinical Chemistry, Harper and Row Publishers, New York, 181.

Henry, R. J. (1979): Colorimeteric methods for determination of serum creatinine. Clinical Chemistry, Principles and techniques, 2nd ed. Harper and Row, pp: 525.

Hoe, C. M. and Harvey, M. (1980): Small animal practice, 2, 109 cited in J.J. Kaneko. Clinical biochemistry of domestic animals, 4th ed. Academic Press.

Holt, J. G.; Krieg, N. R.; Sneath, P. H. A.; staley, J. T. and Williams, S. T. (1996): Bergey's Manual of Determinative Bacteriology, 9th ed. Williams \& Wilkins, Baltimore, Maryland, USA.

Homes, E. L. Jr.; Kwok, T. M. and Mckay, D. G. (1978): The effect of indomethacin on the generalized Schwartzman reaction. Am. J. Pathol. 90: 7.

Howard, A. J., Joseph, T. D.; Bloodworth, L. W.; Forst, J. A.; Chart, H. and Rowe B. (1990): The emergence of ciprofloxacin resistance in Salmonella typhimurium. J. of Antimicrob. Chemotherapy. 26:296-298.

Huang, M. K., Choi, Y. J.; Houde, R.; Lee, J. W.; Lee, B. and Zhao, X. (2004): Effect of Lactobacilli and acidophilic fungus on the production performance and immune responses in broiler chickens. Poult. Sci.:83:788-795.

Jing,W.J.; Shiying,W.G., Hua, W.Y.; Bong,W.K.; Liang, L.B.; Min,W. and Fanxiaoping, X.P. (1998): Effect of yeast polysaccharide on HI antibody level of Newcastle disease and T-cell profileration. Chin. J. Vet. Med. 24 (2): 15-16.

Kaneko, J. J. (1980): Clinical biochemistry of domestic animals, 4th ed., pp. 365-391, Academic Press. Inc., New York, London, Tokyo.

Kheir El-Din, A. W.; Hatem, M. E. and Shouman, M. T. (1986): Experimental investigation on avian Pseudomonas aeruginosa in Egypt. Vet. Med. J. 34 (11): 125-134.

Koncicki, A. and Szubstarska, A. (1988): Role of Pseudomonas aeruginosa in poultry diseases. Veterinarya J. 44 (80): 474-477.

Koneman, E. W.; Allen, S. D.; Schechen, W. M.; Berrjer, P. C. and Winn, W. J. (1992): Color Atlas and Textbook of Diagnostic Microbiology, 4th ed., J. B. Lippincotl Co. Philadelphia, USA

Lilli, R. D. and Fulmen, H. M. A. (1976): Histopathological Techniques and Practical Histopathology, The alauis Division, New York, Acad. Sci. 111: 789-792.

Mankarios, E. (1999): Pharmacological studies on ciprofloxacin in chickens. Ph.D. Thesis, Fac. Vet. Med., Zagazig, Univ., Zagazig, Egypt.

Mazetti, R. (1972): Pseudomoniasis of fowls: Observations and practical considerations. Zooprofilassi, 27 (517): 191-198.

Nakae, M.; Sugahara, Y.; Sasaki, H.; Yasui, H.; Imai, C.; Hasegawa, Y.; Osaka, K. and Shibasaki, K. (1997): Serotypes and drug susceptibility of Pseudomonas aeruginosa isolated from clinical specimens. Jap. J. Antibiotic, 50 (2): 187194.

Natt, M. P. and Herrick, A. C. (1952): A new blood dilunet for counting the erythrocytes and leucocytes of chickens. Poul. Sci. 31: 735-738.
Osama, E. A. R. (1996): Some pharmacological studies ondanofloxacin in chickens. Ph.D. Thesis, Fac. Vet. Med. Zagazig, Univ., Zagazig, Egypt.

Osman, K.; El-Gakee, J. K. A.; Hashaad, M. and Ezz ElDeen, N. A. (1998): Biological characterization of Pseudomonas aeruginosa isolated from diseased chicken. Vet. Med. J. Giza, 46 (4): 451-461.

Ouéré, P. and Girard, F. (1999): Systemic adjuvant effect of cholera toxin in the chicken. Vet. Immunol. Immunopathol. 70: 135-141.

Pascula, M.; Hugas, M.; Badiola J. I.; Monfort, J. M. and Garriga, M. (1999): Lactobacillus salvarius CTC2197 prevents Salmonella enteritidis colonization in chickens, Appl. Environ. Microbiol. 65: 4981-4986.

Pequet, S.; Revoir, S. and Andremont, A. (1990): Faecal excretion of ciprofloxacin after a single oral dose and its effect on faecal bacteria in healthy volunteers. J. Antimicrob. Chemotherapy. 26:125-129.

Quere, P. and Girard, F. (1999): Systemic adjuvant effect of cholera toxin in the chicken. Vet. Immunol. Immunopathol. 70: 135-141.

Quinn, P. J.; Markey, B. K.; Carter, M. E.; Donnelly, W. J. C.; Leonard, F. C. and Maguire, D. (2002): Veterinary Microbiology and Microbial Diseases. Iowa State Univ. Press, Blackwell Science Co., 2121 S. State, Avenue, Ames Iowa 50014-8300, USA.

Raja Rajeswarl, K.; Dhananjaya Reddy, B.; Sharma, B. J. R. and Dhanalakshmi, K. (1992): Normal aerobic bacterial flora of respiratory, intestinal tracts and oviduct of Japanese quails. Ind. Vet. J. 69: $830-883$.

Retiman S. and Framkel, S. (1957): Colorimeteric Method for the determination of serum transaminase activity. Am. J. Clin. Pathol. 28: 65-68.

Ross, J. C.; Christic, G.; Halliday, W. and Jones, R. (1976): Determination of haematology and blood chemistry values in healthy six weeks old broiler birds. Avian Path. 5: 275-281.

Rowe, B.; Ward, L. R. and Trlfall, E. J. (1991): Treatment of multiresistant typhoid fever. Lancet 339: 1422.

Rowe, B.; Ward, L. R. and Trlfall, E. J. (1992): Ciprofloxacin and typhoid fever. Lancet 339: 740.

Schalm, O. W. (1975): Veterinary Haematology. $3^{\text {rd }}$ ed. Bailliere Tindall and Assel Ltd. London.

Schildger, B. J.; Zscock, M. and Gobel, T. (1989): O-Servars and antibiotic of Pseudomonas aeroginosa strains from birds and reptiles. J. Vet. Med. B. 36 (4): 292-296.

Shihata, M. A.; El-Timawy, A. M. and Sedik, I. (1988): Occurrence of Pseudomonas in fowl in upper Egypt. Assiut Vet. Med. J. 20 (39): 169.

Siebert G. (1988): Broad spectrum fluorinated quinolone: Comparison of antibacterial activity with other nalidixic analog. Eur. J. Clin. Microbiol. 2: 548.

Sndecor, G. W. and Cochran, W. G. (1967): Statistical Methods 6th ed., Iowa state Univ. Press, Ames, Iowa, USA.

Tanios, A. I. and Kamel, S. M. (1999): Serovars, antibiogram and virulence feature of Pseudomonas aeruginosa isolated from chickens. Egypt. J. Comp. Pathol. Clin. Pathol.

Van Kempen, E. J. and Zijlstra, W. G. (1961): Colorimeteric determination of haemoglobin. Clin. Chem. Acta. 6: 538.

Younes, J.; Youssef, H.; AbdelKarim, S. and Hassanein, K. (1990): Epidemiological studies of Pseudomonas aeruginosa in chickens, fish and human. Assiut Vet. Med. J. 23 (45): 48-56. 


\section{تجارب علاجية لعدوى بكتريا السودوموناس إيرو جينوزا فى كتاكيت السمان}

أجريت تلك الاراسة لتقييم فعالية طرق العلاج المختلفة باستخدام مستحضرات البروبيوتيك (الخلائق الإحيائية) مثل اللاكتوباسيلاس

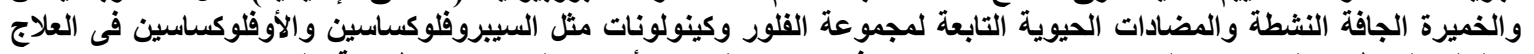

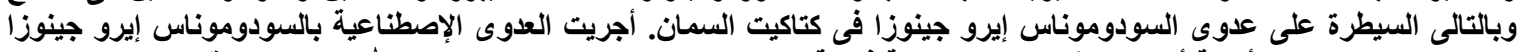

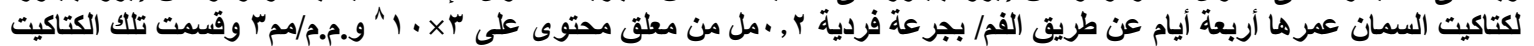

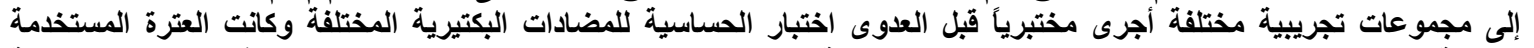

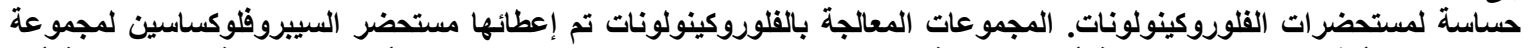

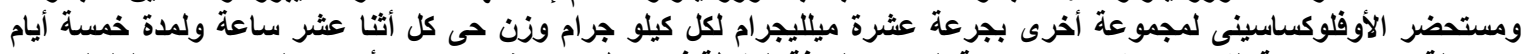

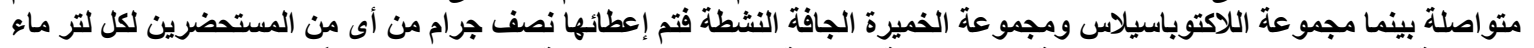

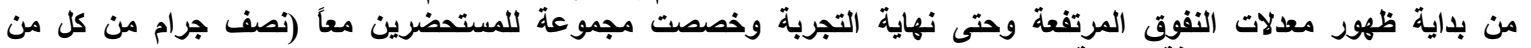

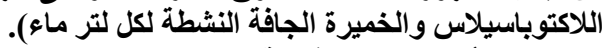

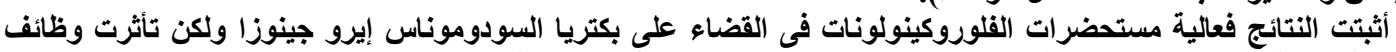

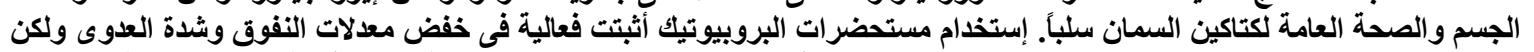

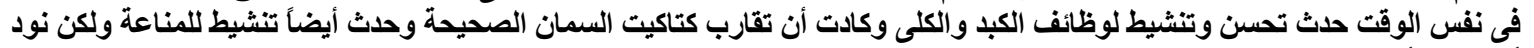

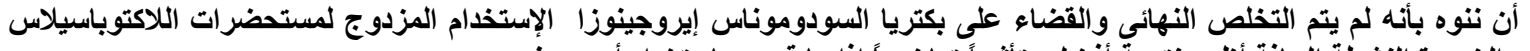

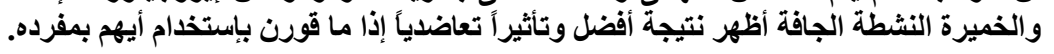

